\title{
EVALUATION OF A DRAG-FREE CONTROL CONCEPT FOR MISSIONS IN LOW EARTH ORBIT
}

\author{
Melissa E. Fleck and Scott R. Starin \\ NASA Goddard Space Flight Center, Greenbelt, MD 20771
}

\begin{abstract}
$\underline{\text { Abstract }}$
Atmospheric drag causes the greatest uncertainty in the equations of motion for spacecraft in Low Earth Orbit (LEO). If atmospheric drag effects can be continuously and autonomously counteracted through the use of a drag-free control system, drag may essentially be eliminated from the equations of motion for the spacecraft. The main perturbations on the spacecraft will then be those due to the gravitational field, which are much more easily predicted. Through dynamical analysis and numerical simulation, this paper presents some potential costs and benefits associated with the fuel used during continuous drag compensation. In light of this cost-benefit analysis, simulation results are used to validate the concept of drag-free control for LEO spacecraft missions having certain characteristics.
\end{abstract}

\section{Background}

Knowledge of spacecraft orbital trajectory, or ephemeris, is vital to all space missions, and in most cases, that knowledge is more useful to meeting the mission objectives when it is highly accurate. Obtaining this knowledge usually involves some empirical measurement of spacecraft trajectory, made on a periodic basis, and propagated between measurements by numerical integration of a dynamical model.

The basic, two-body orbit is not difficult to propagate, but the true trajectory is perturbed from that basic orbit by additional forces with absolute and relative intensities that vary with orbital characteristics. For example, satellites in low Earth orbit (LEO) experience several perturbing forces: predominantly higher order gravitational components, third-body effects, atmospheric drag and solar radiation pressure. The predictability of a LEO satellite trajectory is most severely degraded by the strength and variability of atmospheric drag. ${ }^{1}$ If atmospheric drag could be automatically countered, and therefore removed from the spacecraft equations of motion, considerable improvements in spacecraft ephemeris propagation could be realized. These improvements would, in many cases, translate directly to cost savings in the form of reduced operational staff and a decreased frequency of tracking measurements.

One technology that some missions have implemented for the purpose of automatic drag cancellation is so-called drag-free control. The dragfree satellite was initially proposed in the 1960's and is discussed extensively by Lange. ${ }^{2}$ In such a spacecraft, an unanchored proof mass is enclosed, isolating it from external contact forces such as atmospheric drag and solar radiation pressure. Under ideal conditions, internal disturbance forces may be ignored or mitigated, and the orbit of the proof mass will depend only on gravitational forces. Then, the spacecraft can be forced to follow the orbit of the proof mass by using low-thrust propulsion, thus negating the principle non-gravitational disturbances. With only gravity affecting the drag-free satellite's motion, the propagated trajectory would be much easier to calculate and would remain accurate for much longer periods of time.

To date, all spacecraft that include a drag-free proof mass-successfully launched spacecraft as well as design-phase missions-have had the drag-free aspect as a central facet of their mission., ${ }^{3,4}$ Some use proof masses as a gravitational sensor (e.g. the planned LISA mission-http://lisa.nasa.gov), while others have served as communications beacons in highly predictable orbits (e.g. the Navy TRIAD-1 mission). Because of this central role, the particular requirements of drag-free technology can be a primary design driver affecting every aspect of the mission. Certain materials may be selected to mitigate the disturbances caused by electric or magnetic field gradients, or radiation pressure on the proof mass. The entire configuration of the spacecraft may be tightly constrained and controlled to prevent undesirable differential gravity and self-gravity effects.

So, restrictions on configuration and sensor placement, along with other considerations, make up the natural design path for these drag-free missions. However, it would also be interesting to some mission designers to have access to a more generic drag-free technology, which could be purchased as an off-the-shelf product for missions that use drag-free control as a means to an end, rather than the end in 
itself. Such a drag-free sensor could be constructed to minimize electromagnetic disturbances, and adapted to cancel any gravity gradients in the proof mass enclosure. Generalizing the drag-free sensor could lower the costs incurred by including the sensor, allowing it to be more attractive to a broader range of missions. In particular, any LEO mission could include drag-free control in its design trade space.

This paper represents a generalized trade that would be required of any mission considering the use of a free-floating, protected proof mass as a beacon for guiding a spacecraft along a drag-free trajectory. Part I gives a basic formulation of the motion of the spacecraft-proof mass system. Part II presents a numerical study performed to characterize the potential fuel benefits of making frequent or continuous drag make-up maneuvers. Based on the Part I analysis, the study shows how fuel use depends upon maneuver frequency and spacecraft configuration. Analysis of the resultant fuel costs provides a sense of what mission characteristics would weigh most heavily in the efficacy of drag-free control as a cost-saving device. The study does not purport to be complete; rather, it is hoped that this paper may serve readers as a starting point for their determinations about the potential effectiveness of drag-free technology for their own uses.

\section{Part I: Dynamical Formulation}

To determine the feasibility of using drag-free control on a spacecraft in LEO, it is necessary to estimate the fuel required for continuous drag-free control. That fuel amount can then be compared to the fuel required for traditional periodic orbit correction maneuvers. To keep the analysis general, fuel costs will be represented by cumulative $\Delta \mathrm{V}$. At this point, the attitude of the spacecraft is not taken into consideration. At the end of this section is given the equation to calculate $\Delta \mathrm{V}$ based on the acceleration seen by the drag-free sensor.

The inertial acceleration on a spacecraft, $\ddot{r}_{s}$, is given by:

$$
\ddot{r}_{s}=\sum \ddot{r}_{s g}+\sum \ddot{r}_{s e}-\frac{1}{m_{s}} F_{s p},
$$

where $\ddot{r}_{s g}$ is the acceleration on the spacecraft due to Earth's gravity, $\ddot{r}_{s e}$ is the acceleration due to external perturbative forces (e.g. atmospheric drag, solar radiation pressure, control forces), $m_{s}$ is the spacecraft mass, and $F_{s p}$ is the force of the spacecraft on the proof mass, including gravity. If a proof mass is totally enclosed by a spacecraft, the forces acting on that proof mass will be the sum of the gravitational forces and any interactive forces between the proof mass and the spacecraft:

$$
\ddot{r}_{p}=\sum \ddot{r}_{p \mathrm{~g}}+\sum \ddot{r}_{p e}+\frac{1}{m_{p}} F_{\mathrm{s} p},
$$

where $\ddot{r}_{p}$ is the acceleration of the proof mass, $\ddot{r}_{p g}$ is the acceleration on the proof mass due to Earth's gravitational field, $\ddot{r}_{p e}$ is the acceleration on the proof mass due to external forces other than the spacecraft, and $m_{p}$ is the mass of the proof mass. In order for the spacecraft to follow the proof mass orbit, the total acceleration on the spacecraft must equal that of the proof mass, where the total acceleration on the spacecraft is the spacecraft inertial acceleration plus the acceleration seen by the drag-free sensor:

$$
\ddot{r}_{s}+\ddot{r}_{d f s}=\ddot{r}_{p} .
$$

where $\ddot{r}_{d f s}$ is the acceleration seen by the drag-free sensor. Solving for $\ddot{r}_{d f \mathrm{~s}}$ yields:

$$
\begin{aligned}
& \ddot{r}_{l f s}=\ddot{r}_{p}-\ddot{r}_{s} \\
& =\sum \ddot{r}_{p g}-\sum \ddot{r}_{s g}+F_{s p}\left(\frac{m_{s}+m_{p}}{m_{s} m_{p}}\right)-\sum \ddot{r}_{s e}+\sum \ddot{r}_{p r} .
\end{aligned}
$$

At this point, the assumption will be made that the drag-free sensor is designed such that external accelerations acting on the proof mass, $\ddot{r}_{p e}$, are negligible. In addition, it is assumed that the spacecraft is designed such that mass attraction between the spacecraft and proof mass is also negligible.

\section{Proof Mass Located at Spacecraft Center of Mass}

If the proof mass is located at the spacecraft center of mass, the gravitational terms will cancel out. With the assumptions listed above, the acceleration seen by the drag-free sensor is then reduced to the sum of the external perturbative accelerations acting on the spacecraft:

$$
\ddot{r}_{d s s}=-\sum \ddot{r}_{s e}
$$

\section{Proof Mass Offset from Spacecraft Center of Mass}

A separation between the proof mass and the spacecraft $C G$ can cause a relative acceleration between the spacecraft and proof mass due solely to gravity. The acceleration seen by the drag-free sensor would then be the sum of the external perturbative 
accelerations acting on the spacecraft and the relative acceleration between the spacecraft and proof mass.

The first-order effects of CG offset are readily derived from elementary force-balance principles, but their formulation is dependent upon the spacecraft orbit/attitude profile, e.g. inertially fixed, Earthpointing, etc. Lange provides an approximation for the additional force required to maintain a 1millimeter CG offset. A better understanding of how CG offset would affect a broader range of missions was desired, so expressions were derived for several typical scenarios. Earth-pointing attitude in a circular orbit has been presented here because it represents a very common profile with one of the largest $C G$ offset effects as compared to other orbit/attitude profiles. The following expression describes the firstorder effects of this specific profile:

$$
\Delta a \approx \frac{3 \mu d}{R^{3}} .
$$

Here, $\Delta a$ is the difference in acceleration between the spacecraft and proof mass due only to differential Keplerian motion. The constant $\mu$ is the gravitational parameter, $R$ is the orbit radius, and $d$ is the radial component of the proof mass CG offset. Perfect attitude/orbit maintenance is assumed.

\section{Propulsive Cost of Drag-Free Control}

Once the acceleration seen by the drag-free sensor has been determined, the change in velocity needed to counteract that acceleration at any time during the spacecraft's orbit can then be found by integrating the acceleration with respect to time.

$$
\Delta V=\int \ddot{r}_{d s} d t
$$

Total $\Delta V$ gives a generalized measure of the amount of propulsive effort any maneuver or series of maneuvers will require.

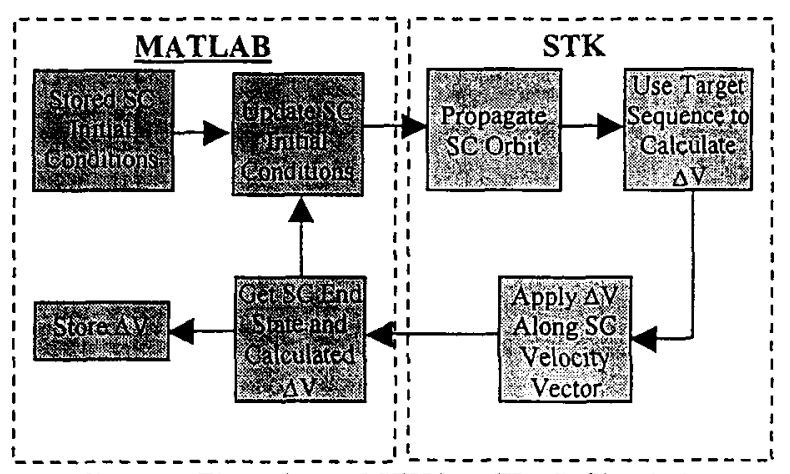

Figure 1: Flow chart of STK/MATLAB Simulation

\section{Part II: Simulation}

The idealized $\Delta V$ required to counteract the effects of atmospheric drag on a spacecraft in low Earth orbit is the integral over time of the acceleration seen by the drag-free sensor (Eq. 7). For this simulation, the assumption is made that the drag-free sensor is located at the spacecraft center of mass, atmospheric drag is the dominant external disturbance force, and internal disturbance forces on the proof mass can be neglected. Of interest is the magnitude of the $\Delta \mathrm{V}$ required for continuous drag compensation on a spacecraft in LEO as compared to the magnitude of the $\Delta \mathrm{V}$ required for periodic drag compensation.

To determine the $\Delta V$ requirements, a simulation was created using a combination of MATLAB and the Astrogator module within Satellite Tool Kit (STK). The Mission Control Sequence (MCS) of the simulation consists of the Spacecraft Initial Conditions, the Propagator, and a Target Sequence. Within the Target Sequence is an Impulsive $\Delta \mathrm{V}$ Maneuver. The Propagator includes two-body gravitational effects and the Jacchia-Roberts atmospheric density model. The simulation, therefore, calculates only the $\Delta \mathrm{V}$ required to counteract atmospheric drag effects. The JacchiaRoberts parameters chosen are daily and average F10.7 values of $150 \mathrm{~W} / \mathrm{m}^{2}$ and a geomagnetic index number of 3.0. The F10.7 values are the mean daily solar flux values at $10.7 \mathrm{~cm}$ wavelength and were chosen to represent an average solar flux. ${ }^{6}$

At the beginning of each simulation, the spacecraft orbital elements are updated using initial conditions stored in the MATLAB script. STK propagates the spacecraft orbit over a time step, $\Delta t$, and uses the Target Sequence to calculate the $\Delta \mathrm{V}$ required, when applied along the spacecraft velocity vector, to raise the spacecraft semi-major axis to within $10 \mathrm{~cm}$ of its original value. Only the velocity vector is targeted because atmospheric drag always acts opposite the spacecraft velocity. The Impulsive Maneuver then applies the $\Delta \mathrm{V}$ along the spacecraft velocity vector. It is assumed at this point that the $\Delta \mathrm{V}$ can be applied exactly along the velocity vector, and therefore there are no losses due to attitude error. MATLAB then takes the spacecraft orbital elements at the end of the Impulsive Maneuver and uses them to update the orbital elements in STK. MATLAB also records the calculated $\Delta \mathrm{V}$. This cycle continues until the spacecraft has been propagated through a total of four weeks. A flowchart of the STK/MATLAB simulation can be seen in Figure 1. 
A total of four parameters affecting drag were varied to establish the different simulation cases studied: orbit altitude and inclination, spacecraft ballistic coefficient, and the time between $\Delta \mathrm{V}$ maneuvers. Table 1 shows a summary of the initial spacecraft parameters used. The ballistic coefficient is the spacecraft mass divided by its cross-sectional area times the drag coefficient. A drag coefficient of 2.0 was assumed for all cases. Values for the ballistic coefficient of the spacecraft were chosen based on information from past spacecraft and are assumed to be constant throughout the four-week propagation $/ \Delta \mathrm{V}$ maneuver cycle (i.e. mass and crosssectional area of the spacecraft are assumed constant)

The time between $\Delta \mathrm{V}$ maneuvers varies from four weeks to one hour. The four-week and two-week time steps are representative of the time between traditional periodic drag compensation maneuvers. The one-hour time step is more representative of continuous drag compensation. Additional time steps are one week, four days, two days, one day, twelve hours, and six hours. These intermediate time steps were simulated to show the general trend in required $\Delta \mathrm{V}$ as the spacecraft gets closer to continuous compensation. Results of the simulations are discussed in the following section.

\section{Simulation Results}

Recall that varying three spacecraft parameters and the time between $\Delta \mathrm{V}$ maneuvers created the different cases of the simulation. A total of 448 cases were run for each of the nine different $\Delta \mathrm{V}$ time steps, for a total of 4032 cases in the simulation. Based on the data, several overall trends can be noted.

The first interesting trend to note is the effect of orbit inclination on the $\Delta V$ cost curve. As can be seen in Figure 2, the initial inclination of the spacecraft orbit appears to have a slight effect on the total $\Delta \mathrm{V}$ required for drag compensation and no effect on the

Table 1: Summary of initial spacecraft parameters for the simulation.

parameters for the simulation.
\begin{tabular}{|c|c|c|}
\hline \multicolumn{3}{|c|}{ Initial Spacecraft Parameters } \\
\hline & & Value/Range \\
\hline Altitude & Varies & $350-700 \mathrm{~km}$ \\
\hline Inclination & Varies & $0-60 \mathrm{deg}$ \\
\hline Ballistic Coefficient & Varies & $25-200 \mathrm{~kg} / \mathrm{m}^{\wedge} 2$ \\
\hline Eccentricity & Fixed & 0 \\
\hline RAAN & Fixed & $0 \mathrm{deg}$ \\
\hline Argument of Perigee & Fixed & $0 \mathrm{deg}$ \\
\hline True Anomaly & Fixed & $0 \mathrm{deg}$ \\
\hline
\end{tabular}

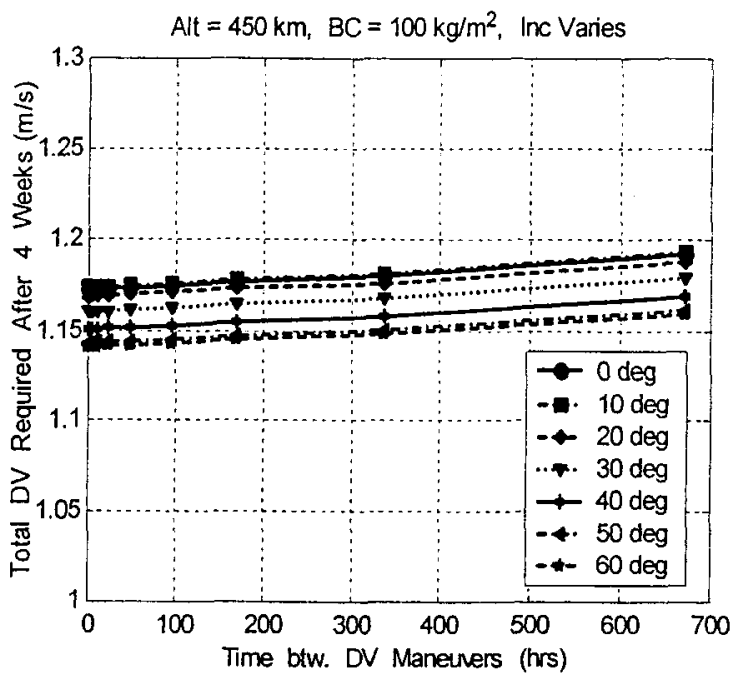

Figure 2: Plot of orbital inclinations at an altitude of 450 $\mathrm{km}$ and a ballistic coefficient of $100 \mathrm{~kg} / \mathrm{m}^{2}$

slope of the $\Delta V$ curve. This trend holds true for all other altitudes and ballistic coefficients studied. This insensitivity to orbit inclination is not surprising considering that drag depends predominantly on spacecraft altitude and exposed area. The slight increase in $\Delta V$ required at low inclinations can be explained in that the Jacchia-Roberts atmospheric density model assumes an increase in average atmospheric density for a given orbital semi-major axis due to the oblateness of the Earth at the equator.

Secondly, in all the cases simulated, there is no extra $\Delta \mathrm{V}$ required for the one-hour $\Delta \mathrm{V}$ maneuvers as compared to the two or four-week maneuvers. In fact, in some cases there is even a significant savings with the one-hour maneuvers. This trend can be seen in Table 2, which shows the ratio of Continuous/Periodic $\Delta V$ required, per year, for drag compensation of a spacecraft at various altitudes and ballistic coefficients. As was pointed out, the inclination of the spacecraft orbit has little effect on

Table 2: Summary of the ratio of Continuous/Periodic $\Delta V$ requirements, per year, for spacecraft drag compensation.

\begin{tabular}{|c|c|c|c|c|c|c|c|c|}
\hline \multicolumn{9}{|c|}{ Ratio of $\Delta V$ Pequirements: Continuous / Periodic Drag Compensation } \\
\hline & \multicolumn{8}{|c|}{ Altitude $(\mathrm{km})$} \\
\hline $\begin{array}{c}\text { Ballistic } \\
\text { Coefficient } \\
\left(\mathrm{kg}^{\prime} \mathrm{m}^{2}\right)\end{array}$ & 350 & 400 & 450 & 500 & 550 & 600 & 650 & 700 \\
\hline 25 & 0.47 & 0.83 & 0.93 & 0.97 & 0.99 & 0.99 & 1.00 & 1.00 \\
\hline 50 & 0.78 & 0.92 & 0.97 & 0.99 & 0.99 & .00 & 1.00 & 1.00 \\
\hline 75) & 0.86 & 0.95 & 0.98 & 0.99 & 1.00 & 1.00 & 1.00 & 1.00 \\
\hline 100 & 0.90 & 0.96 & 0.98 & 0.99 & 1.00 & 1.00 & 1.00 & 1.00 \\
\hline 125 & 0.92 & 0.97 & 0.99 & 0.99 & 1.00 & 1.00 & 1.00 & 1.00 \\
\hline 150 & 0.93 & 0.97 & 0.99 & 0.99 & 1.00 & 1.00 & 1.00 & 1.00 \\
\hline 175 & 0.94 & 0.98 & 0.99 & 1.00 & 1.00 & 1.00 & 1.00 & 1.00 \\
\hline 200 & 0.95 & 0.98 & 0.99 & 1.00 & 1.00 & 1.00 & 1.00 & 1.00 \\
\hline
\end{tabular}


the amount of $\Delta \mathrm{V}$ required; therefore these approximations are valid for all inclinations. The periodic compensation in this calculation is taken to be every four weeks.

As can be seen in Table 2, the cases with the largest $\Delta \mathrm{V}$ savings are at low altitudes and low ballistic coefficients. This result is not surprising since the acceleration due to atmospheric drag increases proportionally with a decrease in ballistic coefficient and increases exponentially with an increase in atmospheric density. The altitude of a spacecraft with a low ballistic coefficient in a low orbit will decrease at a faster rate than a spacecraft with a higher ballistic coefficient in a higher orbit. Therefore, it will require less total $\Delta V$ to raise the semi-major axis after every hour, for four weeks, than it will to raise the semimajor axis at the end of four weeks. At higher ballistic coefficients and higher altitudes, this effect is reduced, and so $\Delta \mathrm{V}$ savings decrease.

At altitudes above $400 \mathrm{~km}$, there does not appear to be any significant difference between the $\Delta V$ required for the four-week maneuver and the $\Delta V$ required for four weeks of one-hour maneuvers. This trend holds true at all inclinations and ballistic coefficients and can be seen in Figure 3. The $\Delta V$ curves for the 4-week time step and 1-hour time step overlap at altitudes over $400 \mathrm{~km}$, indicating little to no extra $\Delta \mathrm{V}$ cost for continuous drag compensation.

Another interesting trend in the data is the effect of the ballistic coefficient on the $\Delta V$ curves. Based on its definition, the ballistic coefficient has a direct effect on the total amount of $\Delta V$ required. The effect

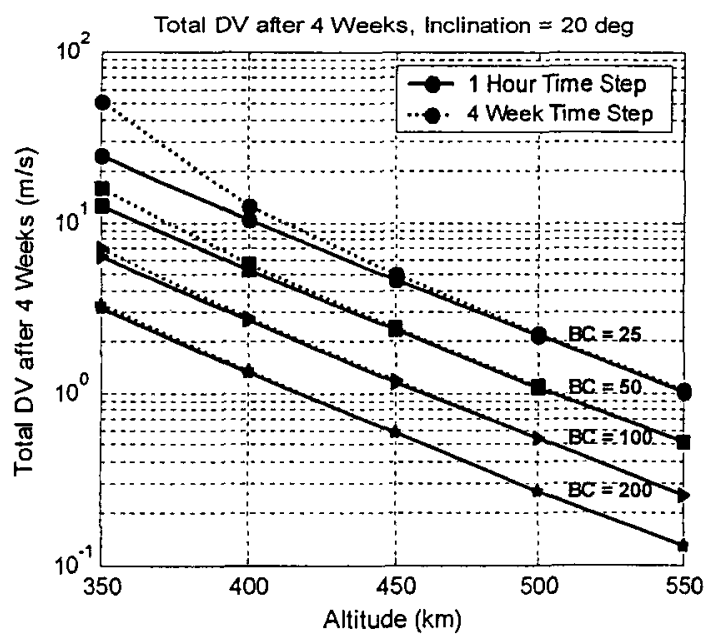

Figure 3: Total $\Delta \mathrm{V}$ after 4 weeks vs. Altitude for various ballistic coefficients. Ballistic coefficients are measured in $\mathrm{kg} / \mathrm{m}^{2}$.
Table 3: Comparison of $\Delta V$ required at different altitudes and ballistic coefficients.

\begin{tabular}{|c|c|c|c|}
\hline \multicolumn{4}{|c|}{$\begin{array}{c}\Delta V \text { Comparis on for Different Ballis tic } \\
\text { Coefficients }\end{array}$} \\
\hline & & & \\
\hline$B C\left(\mathrm{~kg} / \mathrm{m}^{2}\right)$ & \multicolumn{3}{|c|}{1 Hour $\Delta V$ Separation } \\
\hline 25 & 24.64 & 2.13 & 0.13 \\
\hline 100 & 6.19 & 0.53 & 0.03 \\
\hline \multirow[t]{3}{*}{200} & 3.10 & 0.27 & 0.02 \\
\hline & 350 & 500 & 700 \\
\hline & \multicolumn{3}{|c|}{ Altitude $(\mathrm{km})$} \\
\hline \multicolumn{4}{|c|}{ Inclination $=30 \mathrm{deg}$} \\
\hline
\end{tabular}

of a lower ballistic coefficient is an increase in drag. This increase in drag then requires larger amounts of $\Delta V$ to compensate for it. This trend holds true at any altitude and is represented in Table 3.

Of interest is also the effect of the ballistic coefficient on the slope of the $\Delta V$ curve. At altitudes of $400 \mathrm{~km}$ and above, at any inclination, the slopes of the ballistic coefficient curves are approximately equal. This trend can also be seen in Figure 3, and implies that the cost differences between periodic and continuous drag compensation are similar for all ballistic coefficients above $400 \mathrm{~km}$. At lower altitudes, the slopes of the $\Delta \mathrm{V}$ curves vary from one ballistic coefficient to another, because drag lowers the altitude of a spacecraft with a low ballistic coefficient (i.e. a high-drag spacecraft) at a faster rate than a spacecraft with a high ballistic coefficient. A comparison of the $\Delta \mathrm{V}$ cost at lower and higher altitudes can also be seen in Table 3 .

\section{Drag-Free Control Simulation}

From the STK/ MATLAB simulation of the various test cases, it was shown that continuous drag compensation of a spacecraft is feasible from a fuel and orbital maneuvering standpoint. The next step was then to design and test a closed-loop controller to show that drag-free control can be implemented on an arbitrary spacecraft. The goal of the controller is to continuously apply the acceleration required to move the spacecraft such that it follows the proof mass orbit. The drag-free controller and test simulation were created using Simulink, and can be separated into four main parts: spacecraft orbit, proof mass orbit, gap measurement, and the drag-free controller. 
Spacecraft Orbit

As in the STK/MATLAB simulation, the spacecraft in this simulation is assumed to be in a circular, LowEarth Orbit (LEO) with varying inclinations. In addition, the spacecraft is also assumed to be Earth pointing. The spacecraft orbit is calculated in the inertial frame using two-body orbit dynamics with atmospheric drag perturbations. Furthermore, J2 gravitational perturbations can also be included. The effects of $\mathrm{J} 2$ perturbations were also studied, but will be discussed in a future paper. The atmospheric density values used are the average density values listed in [7], with linear interpolation between data points. The total spacecraft acceleration is the sum of the gravitational, drag, and commanded acceleration vectors, and is integrated twice, using a fixed-step, fifth-order Runge-Kutta solver, to obtain the velocity and position of the spacecraft. The integration step size is 5 seconds. The new spacecraft position and velocity vectors are then used to calculate the new gravitational and drag accelerations. The commanded acceleration comes from the drag-free controller.

\section{Proof Mass Orbit}

In the drag-free control system modeled here, the proof mass is assumed to be spherical and enclosed within a small cage or box, however the proof mass itself is not constrained along any axis. The proof mass is allowed to float and orbit freely within its enclosure. Because the proof mass is enclosed within the spacecraft, atmospheric drag does not affect the proof mass orbit. The proof mass orbit, therefore, is modeled using only two-body orbit dynamics. As with the spacecraft orbit, J2 gravitational perturbations can also be included. The total proof mass acceleration is integrated twice to yield the proof mass position and velocity vectors.

\section{$\underline{\text { Gap Measurement }}$}

It is assumed that there exists a sensor (e.g. capacitive or optical) that can measure the gap between the proof mass and any given side of its enclosure. That gap vector is a representation of how far apart the spacecraft and proof mass orbits are. If the proof mass is located at the spacecraft center of mass and the proof mass and spacecraft are following exactly the same orbit, the proof mass will be located at the exact center of the box and the gap vector will be the target gap vector, which, in this system, is assumed to be $\left[\begin{array}{llll}1.0 & 1.0 & 1.0\end{array}\right]^{\mathrm{T}} \mathrm{cm}$.

The gap vector is measured in the spacecraft body frame. It is calculated by first subtracting the proof mass position vector from the spacecraft position vector. Because the spacecraft is assumed to be Earth-pointing, the position vector difference is then converted to the orbit frame, which is assumed to match the body frame. The position vector difference in the orbit (body) frame must then be converted to a gap measurement:

$$
\operatorname{gap}_{m}=\operatorname{gap}_{1}-\left(r_{S_{-} P}^{o}+r_{p_{0}}\right)
$$

where $\operatorname{gap}_{m}$ is the measured gap vector, gap is the target gap vector of $\left[\begin{array}{llll}1.0 & 1.0 & 1.0\end{array}\right]^{\mathrm{T}} \mathrm{cm}, \boldsymbol{r}_{S_{-} P}^{0}$ is the proof mass to spacecraft vector in the orbit frame, and $r_{P O}$ is the offset of the proof mass cage from the spacecraft center of mass.

Because the proof mass is enclosed within a box with a target gap vector of $\left[\begin{array}{llll}1.0 & 1.0 & 1.0\end{array}\right]^{\mathrm{T}} \mathrm{cm}$, the gap along any axis can never be more than $2.0 \mathrm{~cm}$ and never less than $0.0 \mathrm{~cm}$. Otherwise, the proof mass would be outside of the box. Therefore the gap vector initially calculated is fed through logic that limits the measurement to the range $0.0 \mathrm{~cm}$ to $2.0 \mathrm{~cm}$. The vector that exits that logic is then the measurement of the gap vector. At this point in the proof of concept study, drag-free sensor biases due to mass attraction between the proof mass and spacecraft are not included. As the study progresses, those biases, along with other error sources will be introduced.

\section{Drag-Free Controller}

The acceleration needed to keep the spacecraft following the proof mass orbit is calculated with the drag-free controller. The type of controller chosen is a continuous Proportional-Integral-Derivative (PID) controller. The PID gain matrices have not been optimized at this point in the study. Because the goal of this study is to prove the concept of drag-free control for a broad range of LEO spacecraft, the goal of the controller at this point is to simply keep the proof mass from hitting the side of the proof mass cage. The gain matrices were chosen to this end and do not consider optimizing thrust vectors and minimizing the $\Delta \mathrm{V}$ applied.

\section{$\underline{\text { Simulation Scenarios }}$}

To test the effectiveness of the drag-free controller, two different scenarios were established. All cases in the scenarios were run for a four-week period. A comparison of the two scenarios is shown in Table 4. In both scenarios, the attitude of the spacecraft is assumed to be Earth-pointing and perfectly controlled. 


\section{Scenario 1}

Scenario 1 assumes that the center of the proof mass cage is located at the center of mass of the spacecraft and that there are no $\mathrm{J} 2$ or higher order gravitational perturbations acting on the spacecraft or proof mass. Atmospheric drag is the only perturbing acceleration. This scenario is a reflection of the ideal drag-free control situation. The only difference between the spacecraft and proof mass orbits is the atmospheric drag acceleration acting on the spacecraft, which is the effect the drag-free controller is attempting to remove. A total of 64 different cases were run for this scenario. The spacecraft and proof mass altitudes were varied from 350 to $700 \mathrm{~km}$, and the spacecraft ballistic coefficient was varied from 25 to $200 \mathrm{~kg} / \mathrm{m}^{2}$. To reduce computation time, inclination was not varied since it was seen in the STK/MATLAB simulations that differences in inclination have little effect on the spacecraft orbit when $\mathrm{J} 2$ perturbations are not present.

\section{$\underline{\text { Scenario } 2}$}

Scenario 2 assumes that the proof mass cage is offset from the center of mass of the spacecraft. For any given case, the offset is from 1.0 to $10.0 \mathrm{~cm}$ along the velocity, radial, or orbit normal direction. All offsets tend to increase the size of the proof mass orbit. This scenario is a reflection of a more realistic drag-free control situation. As was discussed earlier in this paper, this drag-free control system is not intended to be the spacecraft payload; the spacecraft will not be designed around the drag-free control system. As a result, it is not likely that the proof mass will be located at the spacecraft center of mass. Scenario 2 looks at the effects of offsetting the proof mass on the performance of the drag-free controller. A total of 576 different cases were run for this scenario.

\section{Effects of an Offset Proof Mass}

Between the two scenarios tested, a total of 640 different drag-free control cases were simulated. Due to this large amount of information, it is impossible to discuss all of the results in detail. This section, therefore, attempts to summarize the main trends seen in the scenario results.

A comparison of the results from Scenarios 1 and 2 show that offsetting the proof mass along a single axis can have a considerable impact on the amount of $\Delta \mathrm{V}$ required for continuous drag-free control, depending on the size of the spacecraft, the altitude of its orbit, and the axis along which the proof mass is offset.
Table 4: Summary of initial spacecraft parameters

\begin{tabular}{|c|c|c|c|c|}
\hline \multicolumn{5}{|c|}{ Initial Spacecraft Parameters } \\
\hline Semi-Major Axis & Varies & $\begin{array}{c}350-700 \\
\mathrm{~km}\end{array}$ & Varies & $\begin{array}{c}350-700 \\
\mathrm{~km}\end{array}$ \\
\hline Inclination & Fixed & $20 \mathrm{deg}$ & Fixed & $20 \mathrm{deg}$ \\
\hline Ballistic Coeff. & Varies & $\begin{array}{c}25-200 \\
\mathrm{~kg} / \mathrm{m}^{2}\end{array}$ & Varies & $\begin{array}{c}25-200 \\
\mathrm{~kg} / \mathrm{m}^{2}\end{array}$ \\
\hline Eccentricity & Fixed & 0 & Fixed & 0 \\
\hline RAAN & Fixed & $0 \mathrm{deg}$ & Fixed & $0 \mathrm{deg}$ \\
\hline Arg. of Pe rigee & Fixed & $0 \mathrm{deg}$ & Fixed & $0 \mathrm{deg}$ \\
\hline True Anomaly & Fixed & $0 \mathrm{deg}$ & Fixed & $0 \mathrm{deg}$ \\
\hline Proof Mass Offset & Fixed & $0 \mathrm{~cm}$ & Varies & $1-10 \mathrm{~cm}$ \\
\hline J2 Perturbations & & No & \multicolumn{2}{c|}{ No } \\
\hline
\end{tabular}

When the proof mass is offset from the spacecraft center of mass along the radial or normal directions, the spacecraft and proof mass are placed into slightly different orbits. Figure 5 shows a plot of the percent increase in $\Delta \mathrm{V}$ as the proof mass is offset from the spacecraft center of mass along the radial direction for a spacecraft with a ballistic coefficient of 100 $\mathrm{kg} / \mathrm{m}^{2}$. In the case of a radial offset, the proof mass orbit is larger and there is a differential gravitational acceleration between the two objects. The drag-free control system has to compensate not only for the drag acceleration, but also the differential gravitational acceleration. In addition, for an Earth pointing spacecraft, because the proof mass is actually moving slower than the spacecraft, an additional force is needed on the spacecraft to "catch it up" to the proof mass. The further the proof mass is from the spacecraft center of mass, the greater these effects. In low orbits, the drag acceleration is much larger than the differential gravitational acceleration, and the majority of the $\Delta \mathrm{V}$ required goes to drag compensation. As the altitude becomes higher, the magnitude of the drag acceleration decreases, and the difference between the drag and differential gravitational accelerations becomes smaller. Drag is no longer dominating the differential gravitational acceleration. The majority of the $\Delta \mathrm{V}$ required then goes to compensating for the differential gravitational acceleration. This increase in $\Delta \mathrm{V}$ is also seen as the spacecraft ballistic coefficient becomes larger, since drag acceleration decreases with increasing ballistic coefficient.

For offsets purely along the velocity or normal direction the first order effect of offsetting the proof mass in these directions is a change in true anomaly or inclination, respectively. A true anomaly offset 


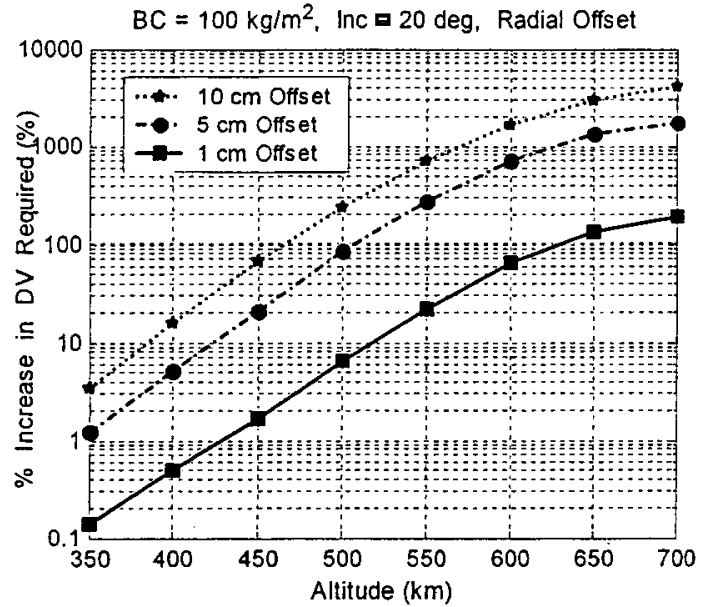

Figure 5: Percent increase in $\Delta \mathrm{V}$ required for drag compensation as the proof mass is offset from the spacecraft center of mass in the radial direction.

requires no additional $\Delta \mathrm{V}$ for continuous drag compensation. In the case of a true anomaly difference, the proof mass and spacecraft are still in the same orbit and thus have the same gravitational acceleration. As with the non-offset proof mass, drag acceleration on the spacecraft is the only difference between the proof mass and spacecraft."

Like a change in proof mass orbit radius, a change in proof mass inclination puts the proof mass and spacecraft into slightly different orbits. This will require additional $\Delta \mathrm{V}$ to maintain the relative distance between the spacecraft center of mass and the proof mass which can be seen in Figure 6.

In addition to the effects discussed above, for a rotating spacecraft, there is also an acceleration due to the rotation of the spacecraft. Since this study does not consider spacecraft attitude, that rotational acceleration is not accounted for.

In both Scenarios 1 and 2, the drag-free controller does a good job of compensating for drag and keeping the spacecraft orbit close to its initial conditions. In all cases tested, the semi-major axis is maintained to within about $25 \mathrm{~cm}$.

\footnotetext{
* The second order effects of a velocity offset are interesting and are being investigated further. They will be discussed in a future paper.
}

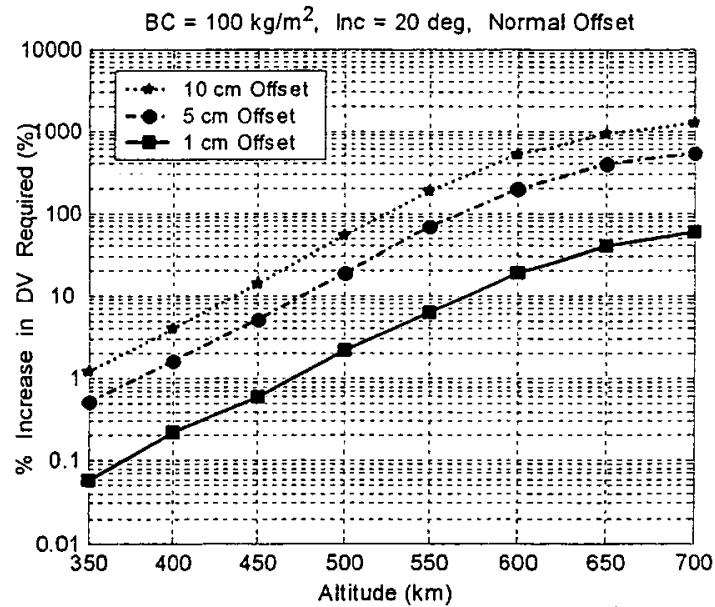

Figure 6: Percent increase in $\Delta V$ required for drag compensation as the proof mass is offset from the spacecraft center of mass in the normal direction.

\section{Conclusion}

An STK/MATLAB simulation compares the $\triangle \mathrm{V}$ cost of maintaining a spacecraft in LEO using periodic orbit maneuvers to the $\Delta \mathrm{V}$ cost of using continuous drag compensation. In terms of $\Delta \mathrm{V}$ cost, continuous drag compensation of a spacecraft in LEO is a viable means of spacecraft orbit maintenance. $\Delta \mathrm{V}$ savings are most significant for spacecraft with low ballistic coefficients in low orbits. Above altitudes of about $450 \mathrm{~km}$, there is little difference between $\Delta V$ costs for continuous and periodic drag compensation for spacecraft of any size. At altitudes of $450 \mathrm{~km}$ and below, spacecraft with ballistic coefficients above $100 \mathrm{~kg} / \mathrm{m}^{2}$ show little or no extra $\Delta \mathrm{V}$ cost for continuous drag compensation.

Once the feasibility of continuous drag compensation was determined, Simulink was used to design and test the proof mass-based drag-free controller concept, simulating various spacecraft in various orbits. In addition, the effects of an offset proof mass on controller performance were also investigated.

The performance of the drag-free controller when subjected to only drag perturbations is excellent. The controller is able to closely maintain the original orbit of the spacecraft. When the proof mass cage is offset from the spacecraft center of mass, the controller performance degrades slightly. However, the real drawback to the offset proof mass is seen in $\Delta \mathrm{V}$ cost.

To first order, there is no difference in the amount of $\Delta \mathrm{V}$ required to compensate for drag when the dragfree sensor is offset along the velocity direction . 
However, when the sensor is offset $10 \mathrm{~cm}$ in the radial direction (i.e. vertically), the cost of maintaining the drag-free orbit can increase by as much as a factor of 50 (see Figure 5). If the offset is $10 \mathrm{~cm}$ in the normal direction, $\Delta \mathrm{V}$ costs still can increase by a factor of 15 (Figure 6). For any given spacecraft configuration, there is an altitude at which it becomes just as expensive to compensate for proof mass offset as it does to compensate for drag.

Figure 7 shows the relationship between altitude and acceleration-both for drag accelerations at given ballistic coefficients, and for center-of-mass offset effect for given radial offsets. The figure demonstrates cross-over altitudes where the control acceleration required for drag compensation equals the acceleration required to maintain the center-ofmass offset. The results of the simulation verify that there is an upper bound on altitudes for which dragfree control is feasible from a fuel use perspective. The closer the proof mass can be placed to the spacecraft center of mass, the higher that bound. That bound will also be higher if the proof mass offset can be controlled to a simple true anomaly offset. Although rotational accelerations are not taken into account in this study, they will also contribute to the overall effects when the proof mass cage is offset from the spacecraft center of mass.

\section{References}

${ }^{1}$ Wertz, James R. 2001. Mission Geometry: Orbit and Constellation Design and Management, $\mathrm{El}$
Segundo, CA, and Dordrecht, The Netherlands: Microcosm Press and Kluwer Academic Publishers.

2 Lange, Benjamin, "The Drag Free Satellite," AIAA Journal, Vol. 2, No. 9. Sept. 1964, pp. 1590-1606.

${ }^{3}$ Staff of the Space Department, The Johns Hopkins University Applied Physics Laboratory and Staff of the Guidance and Control Laboratory, Stanford University, "A Satellite Freed of All But Gravitational Forces: TRIAD-I," Journal of Spacecraft and Rockets, Vol. 1, No. 9, Sept. 1974, pp. 637-644.

${ }^{4}$ Eisner, A. and S. M. Yionoulis, "NOVA-1 - The 'Drag-Free' Navigation Satellite," Proceedings from the 1982 Aerospace Meeting, NASA Ames Research Center, Moffett Field, CA: 24-25 March 1982, pp. 26-31.

5 Vaillon, L, J. Borde, T. Duhamel, and P. Damilano, "Drag-Free Control Systems and Technologies," Space Technology, Vol. 16, No. 5/6, pp. 245-254.

${ }^{6}$ Vallado, David A. 2001. Fundamentals of Astrodynamics and Applications, $2^{\text {nd }}$ edition. El Segundo, CA, and Dordrecht, The Netherlands: Microcosm Press and Kluwer Academic Publishers.

${ }^{7}$ Wertz, James R. and Wiley J. Larson, eds. Space Mission Analysis and Design, $3^{\text {rd }}$ edition. El Segundo, CA, and Dordrecht, The Netherlands: Microcosm Press and Kluwer Academic Publisher

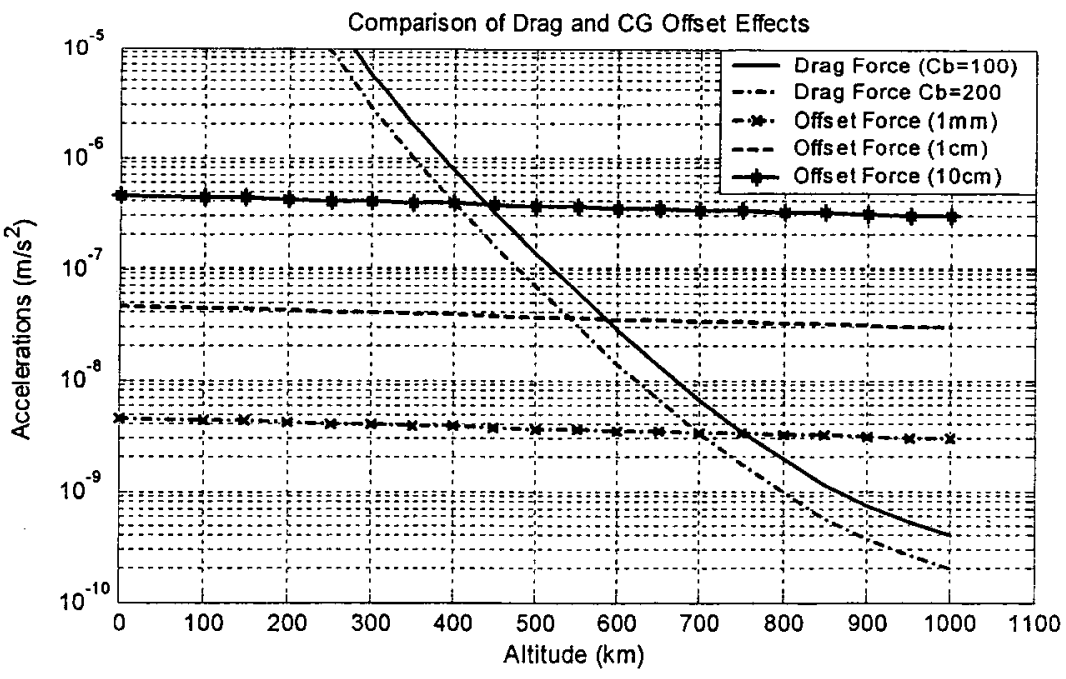

Figure 7: Drag accelerations for spacecraft with given ballistic coefficients as compared with accelerations required to compensate for several given radial offsets of the drag-free proof mass from the spacecraft center of mass. 